\title{
Transition Metal Migration Can Facilitate Ionic Diffusion in Defect Garnet Based Intercalation Electrodes
}

\author{
Nicholas H. Bashian, ${ }^{\dagger}$ Samantha Abdel-Latif, ${ }^{\dagger}$ Mateusz Zuba, ${ }^{\ddagger}$ Kent J. Griffith, ${ }^{\top}$ Alex M. \\ Ganose,$^{\S}$ Joseph W. Stiles, ${ }^{\dagger}$ Shiliang Zhou, ${ }^{\dagger}$ David O. Scanlon, ${ }^{\S}, \|, \perp, \#$ Louis F. J. Piper, ${ }^{\ddagger} @$ and \\ Brent C. Melot*,† \\ $\dagger$ Department of Chemistry, University of Southern California, Los Angeles, CA 90089, USA \\ $\ddagger$ Department of Physics, Applied Physics and Astronomy, Binghamton University, Binghamton, New York \\ 13902, United States \\ \Department of Chemistry, University of Cambridge, Lensfield Road, Cambridge CB2 1EW, United \\ Kingdom \\ $\S$ Department of Chemistry, University College London, 20 Gordon Street, London WC1H 0AJ, U.K. \\ ||Diamond Light Source Ltd., Diamond House, Harwell Science and Innovation Campus, Didcot, \\ Oxfordshire OX11 ODE, U.K. \\ $\perp$ Thomas Young Centre, University College London, Gower Street, London WC1E 6BT, U.K. \\ \# The Faraday Institution, Quad One, Harwell Science and Innovation Campus, Didcot, U.K. \\ @ Materials Science 83 Engineering, Binghamton University, Binghamton, New York 13902, United States \\ E-mail: melot@usc.edu
}




\section{Supporting Information}

\section{Experimental Details}

Synthetic Methods. $\mathrm{Y}_{2}\left(\mathrm{MoO}_{4}\right)_{3}$ was synthesized using a modified sol-gel method. Solutions of stoichiometric amounts of $\mathrm{Y}\left(\mathrm{NO}_{3}\right)_{3} \times 6 \mathrm{H}_{2} \mathrm{O}$ and $\mathrm{Na}_{2} \mathrm{MoO}_{4} \times 2 \mathrm{H}_{2} \mathrm{O}$ were prepared and stirred together. After centrifugation, a white precipitate was isolated from the solution using diethyl ether. The precipitate was then dried under vacuum, ground into a powder, and washed with DI water. The clean powder was calcined in a tube furnace at $900{ }^{\circ} \mathrm{C}$ under nitrogen for 5 hours after a 6 hour heating ramp. Upon cooling, this yielded a whitish-gray powder that was ground via mortar and pestle.

$\mathrm{Al}_{2}\left(\mathrm{MoO}_{4}\right)_{3}$ was synthesized by combining stoichiometric amounts of $\mathrm{Al}_{2} \mathrm{O}_{3}$ and $\mathrm{MoO}_{3}$. Powders were ground together via mortar and pestle and pressed into pellets. The resulting pellets was placed in a crucible and heated for 40 hours at $650{ }^{\circ} \mathrm{C}$, with a 5 hour heating ramp. The compound was subsequently cooled to ambient temperature over a two-hour period and ground to yield a white powder

Operando Materials Characterization. Operando XRD patterns were collected at the Advanced Photon Source (APS), Argonne National Laboratory using the AMPIX electrochemical cell, following the method detailed by Borkiewicz et al. ${ }^{1}$ In brief, electrochemical cells equipped with glassy carbon windows were prepared using free standing film electrodes, glass fiber separators soaked in electrolyte solution, and Li foil counter electrodes. High resolution synchrotron powder diffraction data was collected using beamline 17-BM at the APS, Argonne National Laboratory using an average wavelength of $0.24116 \AA$, with a Perkin-Elmer 2D plate detector. XRD collection was performed in transmission geometry through the cell windows. GSAS-II software was used to integrate patterns into the intensity vs. $2 \theta$ format displayed. ${ }^{2}$ Operando X-ray Absorption Spectroscopy (XAS) measurements were performed at beamline 12-BM, APS using the AMPIX electrochemical cell, equipped with a glass fiber separator and a Li foil combined counter and reference electrode. XAS measurements were performed in transmission geometry at the Mo K-edge $(20.0 \mathrm{KeV})$, using Mo foil as a reference. Scans were collected in transmission mode over a span of 19 minutes with a constant interval of 120 minutes between scans. XAS data proccessing was carried out using the ATHENA software of the package IFEFFIT. ${ }^{3}$ The EXAFS component was normalized and converted to wavenumber. The resultant signal in k-space was multiplied with a $\mathrm{k}^{3}$, Fourier-transformed and left with no phase shift correction. All displayed EXAFS data is shown with no phase shift correction. Ab initio calculations on relevant structure models were done using the code FEFF8.2 with fits being performed using the ARTEMIS software of the IFEFFIT package. ${ }^{3,4}$

Solid State NMR Measurements. Solid-state nuclear magnetic resonance (NMR) spectroscopy was performed on a Bruker Advance III spectrometer at a magnetic field strength of $16.4 \mathrm{~T}$ corresponding to Larmor frequencies $\left(\nu_{0}\right)$ for ${ }^{7} \mathrm{Li}(\mathrm{I}=3 / 2),{ }^{27} \mathrm{Al}(\mathrm{I}=5 / 2),{ }^{89} \mathrm{Y}(\mathrm{I}=1 / 2)$, and ${ }^{95} \mathrm{Mo}(\mathrm{I}=5 / 2)$ of $272.1 \mathrm{MHz} 182.4 \mathrm{MHz}, 34.3 \mathrm{MHz}$, 
and 45.6 MHz, respectively. ${ }^{7} \mathrm{Li}$ and ${ }^{27} \mathrm{Al}$, spectra were collected with a $3.2 \mathrm{~mm} \mathrm{HXY}$ Bruker probe under magic angle spinning (MAS) at a rate of $20 \mathrm{kHz} .{ }^{89} \mathrm{Y}$ and ${ }^{95} \mathrm{Mo}$ spectra were recorded in a $4.0 \mathrm{~mm}$ HXY Bruker probe at ambient temperature and an MAS rate of $12.5 \mathrm{kHz}$. All samples were packed in $\mathrm{ZrO}_{2}$ rotors with a Kel-F cap; lithiated samples of 8-18 mg were center-packed in the rotor between poly(tetrafluoroethylene) ribbon. Single-pulse measurements were performed on all samples; rotor-synchronized Hahn echo spectra were measured alongside the single-pulse spectra for ${ }^{7} \mathrm{Li}$ and ${ }^{27} \mathrm{Al}$. Excitation pulses of $\pi / 6$ were used for ${ }^{27} \mathrm{Al}$ and ${ }^{95} \mathrm{Mo}$ to ensure homogeneous excitation of sites with variable nuclear quadrupolar coupling constants. Spectra were referenced as follows: ${ }^{7} \mathrm{Li}-1.0$ $\mathrm{M} \mathrm{LiCl}($ aq. $)$ at $0 \mathrm{ppm} ;{ }^{27} \mathrm{Al}-\mathrm{AlF}_{3}$ at $-15 \mathrm{ppm} ;{ }^{89} \mathrm{Y}-\mathrm{Y}_{2} \mathrm{O}_{3}$ at 273 and $314 \mathrm{ppm} ;{ }^{95} \mathrm{Mo}-1.0 \mathrm{M} \mathrm{Na}_{2} \mathrm{MoO}_{4}$ (aq.) at $0 \mathrm{ppm}$. Recycle delays were $15 \mathrm{~s}$ for ${ }^{7} \mathrm{Li}, 25 \mathrm{~s}$ for ${ }^{27} \mathrm{Al}, 400 \mathrm{~s}$ for ${ }^{89} \mathrm{Y}$, and $10 \mathrm{~s}$ for ${ }^{95} \mathrm{Mo}$. The $T_{1}$ relaxation of ${ }^{7} \mathrm{Li}$ and ${ }^{27} \mathrm{Al}$ were checked to ensure the spectra were quantitative (recycle delay $\geq 5 T_{1}$ ). $T_{1}$-filtered ${ }^{7} \mathrm{Li}$ measurements were performed with a recycle delay of $0.1 \mathrm{~s}$.

First principles calculations of the NMR shift and quadrupolar tensors were performed in the density functional theory-based CASTEP code with the Perdew Burke and Ernzerhof (PBE) exchange-correlation functional. ${ }^{5,6}$ Geometry optimization of the starting structures was performed by relaxing the (i) atomic positions or (ii) atomic positions and lattice parameters until the maximum force was less than $0.01 \mathrm{eV} \cdot \AA^{-1} \cdot{ }^{7,8}$ Full shift and quadrupolar tensor calculations were performed on the unrelaxed structure and both relaxed structures. Geometry optimization and NMR calculations used Vanderbilt ultrasoft pseudopotentials calculated "on-the-fly" in CASTEP with a plane wave basis set, plane wave energy cut-off of $700 \mathrm{eV}$, and Brillouin zone sampling with a Monkhorst-Pack grid of k-points finer than $0.04 \times 2 \pi \AA^{-1} \cdot{ }^{9,10}$ Core electrons for NMR calculations were reconstructed with the gauge-including projector-augmented wave (GIPAW) method implemented in the code.

Electrochemical Characterization. The electrochemical performance of the as-prepared materials was characterized using Swagelok-type cells assembled in an argon-filled glovebox, using Li metal as a combined counter and reference electrode and Whatman GF/D borosilicate glass fiber sheets as the separator. $1 \mathrm{M} \mathrm{LiPF}_{6}$ in ethylene carbonate and dimethylcarbonate $(1: 1 \mathrm{w} / \mathrm{w})$ was used as the electrolyte (LP30). Powders of active material were dried under vacuum to remove any residual moisture. Thick film electrodes were prepared by blending $10 \%$ graphite powder (300 mesh), 10\% acetylene black, 20\% polytetrafluoroethylene (average particle size of $1 \mu \mathrm{m}$ ), and $60 \%$ active material, and pressed under a hydrostatic pressure of 0.9 tons, with a typical electrode mass of 20 mg. All cell components and electrodes were dried under vacuum at $110{ }^{\circ} \mathrm{C}$ for 1 hour before assembly. Galvanostatic cycling was typically performed from $1 \mathrm{~V}-3.5 \mathrm{~V}$ at a current corresponding to a $\mathrm{C} / 20$ rate, while cyclic voltammetry was collected with a sweep rate of $0.1 \mathrm{mV} / \mathrm{s}$. Operando XRD patterns and operando XAS spectra were collected using an AMPIX electrochemical cell equipped with two glassy carbon windows. The same glass fiber separators, metallic counter electrodes, and electrolyte solutions described previously were used during all operando experiments. 
Computational Methodology. First-principles calculations were performed within the framework of Density Functional Theory using the Vienna ab initio Simulation Package (VASP). ${ }^{11-14}$ Interactions between core and valence electrons were described using the Projector Augmented Wave method. Convergence with respect to the plane wave basis set and $k$-point sampling were tested, with a cut-off energy of $400 \mathrm{eV}$ and $k$-point meshes of $\Gamma$-centred $1 \times 2 \times 1$ and $1 \times 2 \times 2$ found to be sufficient for $\mathrm{Al}_{2}\left(\mathrm{MoO}_{4}\right)_{3}$ and $\mathrm{Y}_{2}\left(\mathrm{MoO}_{4}\right)_{3}$, respectively. Geometry optimisations were performed using the PBEsol functional, ${ }^{15}$ a version of the Perdew Burke and Ernzerhof (PBE) functional ${ }^{6}$ revised for solids. PBEsol has previously been shown to reproduce the lattice parameters for a broad range of oxide systems. ${ }^{16,17}$ Further computational details on the accuracy of the structural optimizations are given in the Supporting Information Figures S16, S15, and Table T3. Density of states and band structure diagrams were plotted using the sumo package. ${ }^{18}$

In order to correctly describe the electronic structure of the materials, the hybrid functional, HSE06, ${ }^{19}$ was employed for band structure and density of states calculations. HSE06 combines $75 \%$ exchange and $100 \%$ of the correlation energies from PBE together with $25 \%$ exact Hartree-Fock (HF) exchange at short ranges and has been shown to perform well for oxide materials. ${ }^{16}$ 
Change of Intensity in XRD. As shown in Figure S1, the intensity of diffracted peaks is greatly reduced upon lithiation of $\mathrm{Al}_{2}\left(\mathrm{MoO}_{4}\right)_{3}$. Conversely, the diffuse background increases, indicating an increase in incoherent scattering from amorphous regions. Similar behavior is observed in $\mathrm{Y}_{2}\left(\mathrm{MoO}_{4}\right)_{3}$ where peaks uniformly loose intensity.

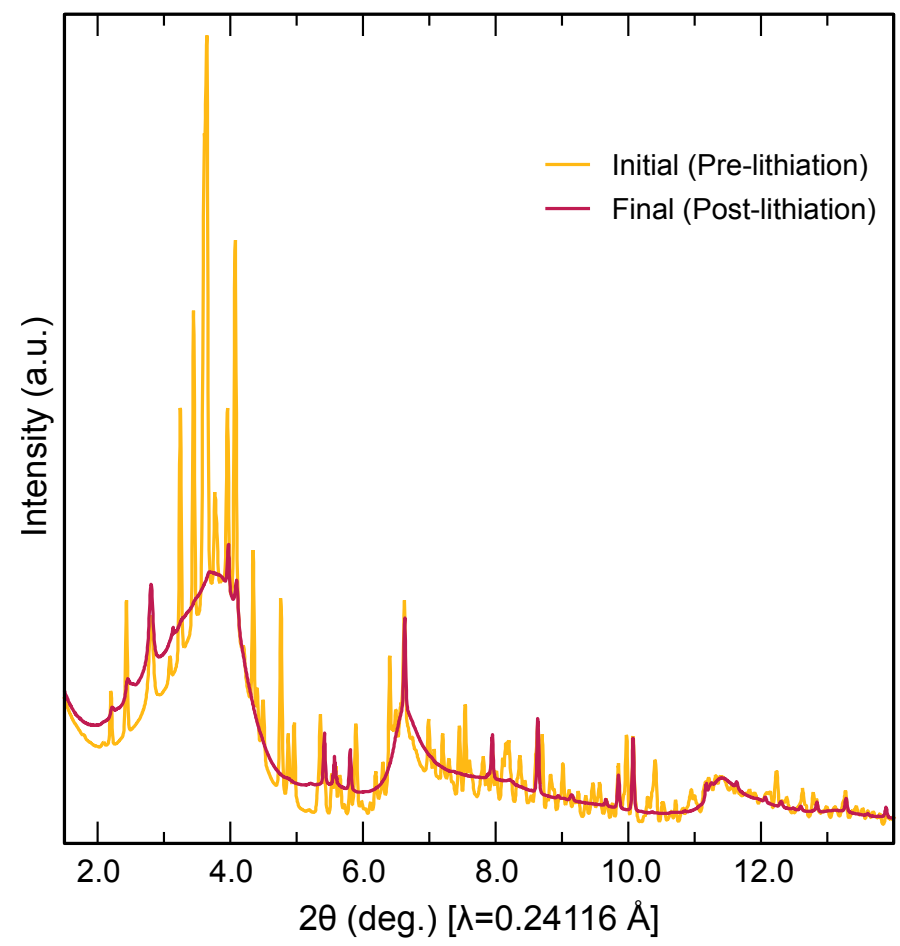

Figure S1: Upon lithiation, an increase in the diffuse background and a decrease in peak intensity is observed in $\mathrm{Li}_{x} \mathrm{Al}_{2}\left(\mathrm{MoO}_{4}\right)_{3}$ which is associated with the formation of amorphous products. 
Cyclic Voltammetry of $\mathbf{L i}_{x} \mathbf{A l}_{2}\left(\mathrm{MoO}_{4}\right)_{3}$. Figure S2 demonstrates the large irreversible capacity associated with the initial lithiation of $\mathrm{Li}_{x} \mathrm{Al}_{2}\left(\mathrm{MoO}_{4}\right)_{3}$. A substantial decrease in the charge passed is observed between the first and second reductive cycles, while the broad featureless peaks are maintained.

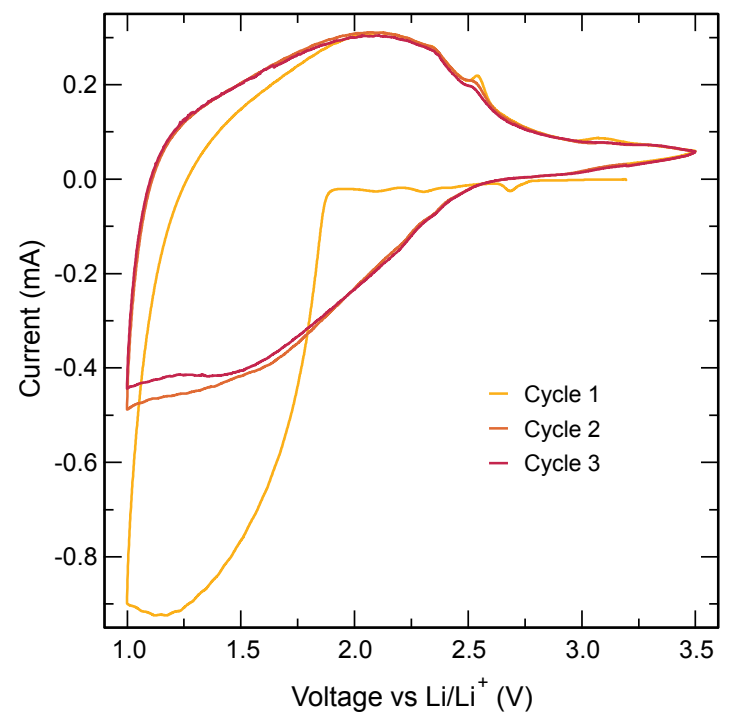

Figure S2: Cyclic voltammogram of $\mathrm{Al}_{2}\left(\mathrm{MoO}_{4}\right)_{3}$ with 3 cycles against Li metal in a two electrode Swagelok cell. The first cycle differs substantially from the following cycles.

Cyclic Voltammetry of $\mathbf{L i}_{x} \mathbf{Y}_{2}\left(\mathbf{M o O}_{4}\right)_{3} \cdot \quad \mathrm{Li}_{x} \mathrm{Y}_{2}\left(\mathrm{MoO}_{4}\right)_{3}$ has more Faradaic behavior as seen in Figure S3 which shows the sharper peaks present in a cyclic voltammogram. The intercalation based charge storage, and associated polyhedral rotations result in sharper features and less broad peaks, when compared to Figure S2.

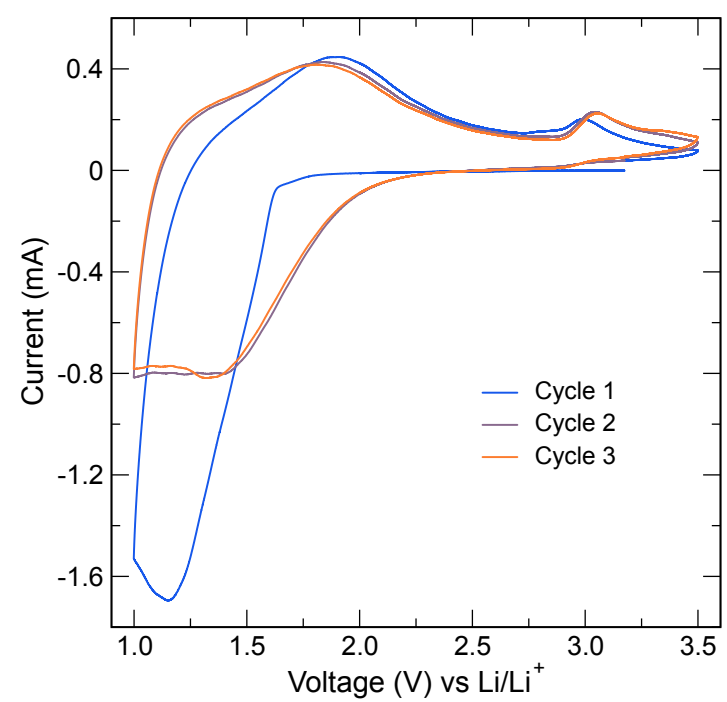

Figure S3: $\mathrm{Y}_{2}\left(\mathrm{MoO}_{4}\right)_{3}$ cyclic voltammogram shows Faradaic peaks associated with lithation. The first cycle shows a larger area due to irreversibility. 
Y K-edge XAS Measurements. The Y K-edge was measured on a series of samples of $\mathrm{Li}_{x} \mathrm{Y}_{2}\left(\mathrm{MoO}_{4}\right)_{3}$ and the radial distribution was calculated from the Fourier transform of the k-space data, in Figure S4 and S5 respectively. In the radial distribution function, peaks in higher shells are seen to change as the structure is rearranged during ion insertion. The displayed data is not phase shifted.

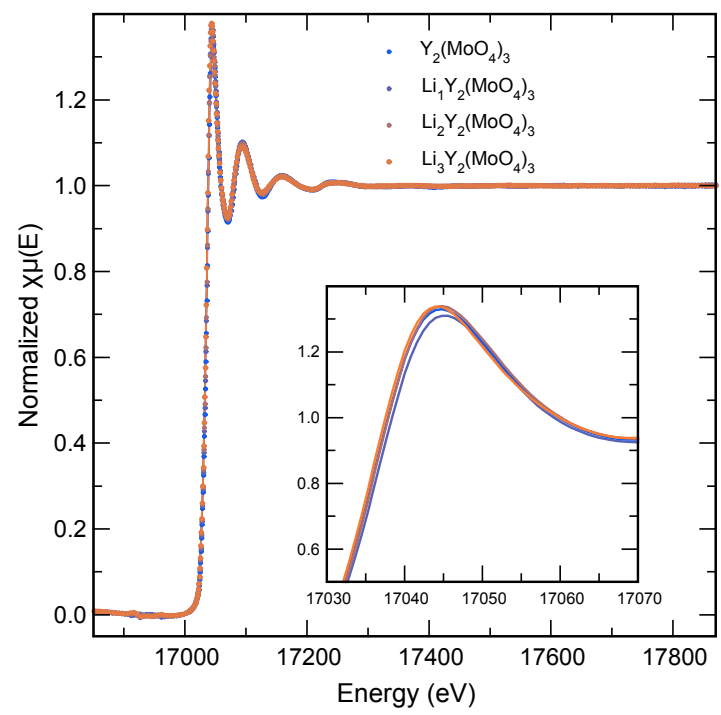

Figure S4: XAFS measurements on the $\mathrm{Y}$ K-edge in ex-situ samples of $\mathrm{Li}_{x} \mathrm{Y}_{2}\left(\mathrm{MoO}_{4}\right)_{3}$ show a constant K-edge energy despite different states of charge.

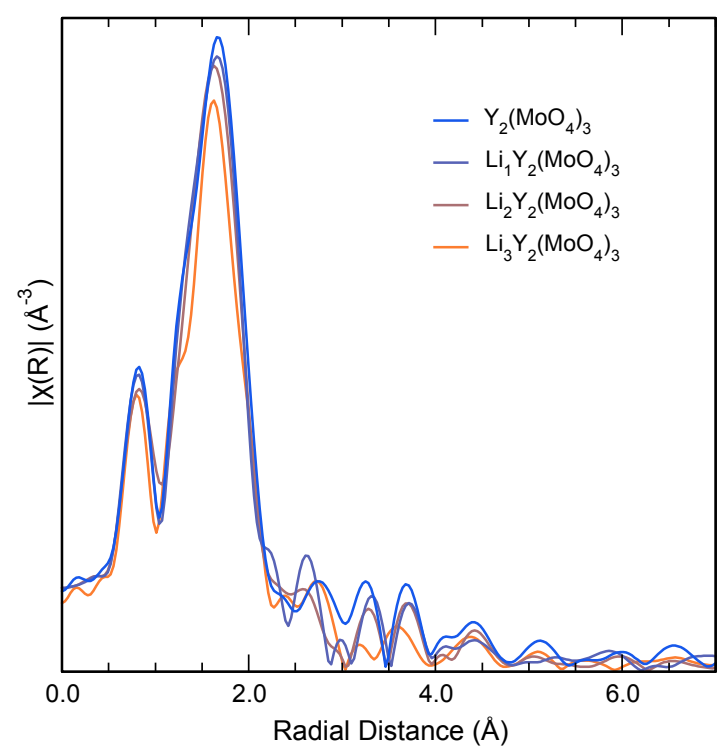

Figure S5: The radial distribution function as obtained from the Fourier transform of Y K-edge data collected on $\mathrm{Y}_{2}\left(\mathrm{MoO}_{4}\right)_{3}$. The peak at $1.8 \AA$ represents the $\mathrm{Y}$-O distance within the $\mathrm{YO}_{6}$ octahedra and remains constant at various states of charge. 
Comparison of Mo K-edge to Y K-edge XAS Measurements. The Y K-edge was measured for a series of $\mathrm{Y}_{2}\left(\mathrm{MoO}_{4}\right)_{3}$ samples at various states of lithiation, which demonstrated that the $\mathrm{Y}$ K-edge did not shift due to the redox inactivity of $\mathrm{Y}$ in this structure. For comparison, the Mo K-edge was measured on the same set of samples, after the Y K-edge measurements. As shown in figure S6, the Mo K-edge showed a substantial shift and restructuring, analogous to that which was observed in the operando measurements performed on $\mathrm{Y}_{2}\left(\mathrm{MoO}_{4}\right)_{3}$. Operando measurements were not performed on the Y K-edge due to beam time constraints.
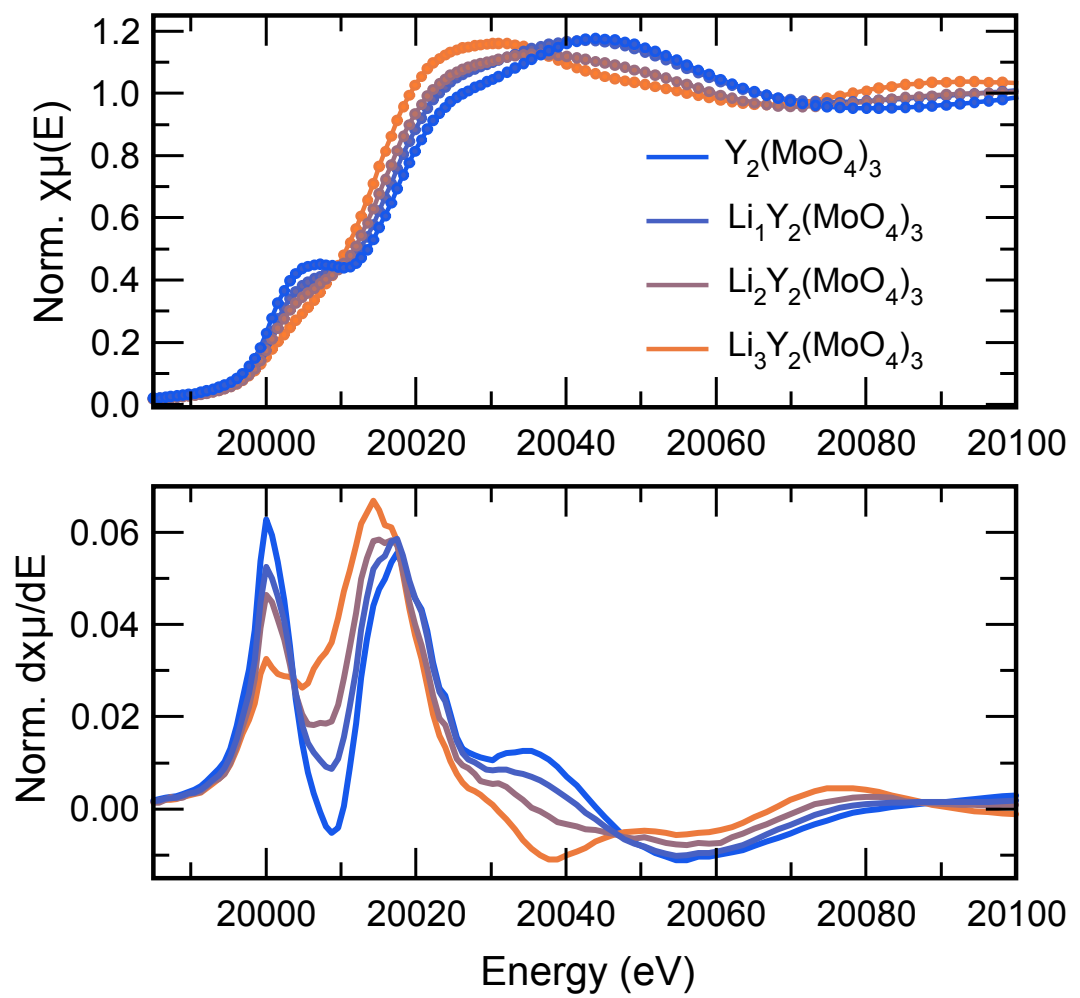

Figure S6: The Mo K-edge in $\mathrm{Li}_{x} \mathrm{Y}_{2}\left(\mathrm{MoO}_{4}\right)_{3}$ shows a shift to lower energies corresponding to the reduction of $\mathrm{Mo}^{6+}$ during discharge. This change matches the results seen in operando measurements on the same system. 
EXAFS Radial Distribution Fits. Radial distribution fits were performed on Mo K-edge EXAFS data of the pristine and fully lithiated endpoints of both $\mathrm{Al}_{2}\left(\mathrm{MoO}_{4}\right)_{3}$ and $\mathrm{Y}_{2}\left(\mathrm{MoO}_{4}\right)_{3}$. Fits of the pristine $\mathrm{Y}_{2}\left(\mathrm{MoO}_{4}\right)_{3}$ and $\mathrm{Al}_{2}\left(\mathrm{MoO}_{4}\right)_{3}$ agreed well with their starting structure, as shown in Figure S7(a) and S7(c) respectively. It was seen that the initial $\mathrm{Al}_{2}\left(\mathrm{MoO}_{4}\right)_{3}$ structure decayed upon lithiation, while new scattering lengths increased in intensity throughout discharge. As the EXAFS data is compromised mainly of the first atomic shell about the Mo atoms, this change in signal represents a marked rearrangement in the structure. This rearrangement represents a transition from the initial tetrahedral $\mathrm{MoO}_{4}$ environment to an octahedral geometry $\left(\mathrm{MoO}_{6}\right)$ about the scattering Mo atoms, which results in an elongation of the Mo-O bonds. It is clear that the rearrangement induced by lithiation of $\mathrm{Al}_{2}\left(\mathrm{MoO}_{4}\right)_{3}$ leads to the formation of new species, as Mo migrates from the tetrahedral site. In comparison, the lithiation of $\mathrm{Y}_{2}\left(\mathrm{MoO}_{4}\right)_{3}$ leads to more nuanced changes, especially in the first shell region centered around $1.25 \AA$ as shown in Main Text Figure 4(c). It was found that EXAFS radial distribution data of the fully lithiated $\operatorname{Li}_{6} \mathrm{Y}_{2}\left(\mathrm{MoO}_{4}\right)_{3}$ structure, while more disordered, fits well with the structure of the pristine material, as shown in Figure S7(b). In $\mathrm{Y}_{2}\left(\mathrm{MoO}_{4}\right)_{3}$, the Mo local environment is better maintained throughout the lithiation process.
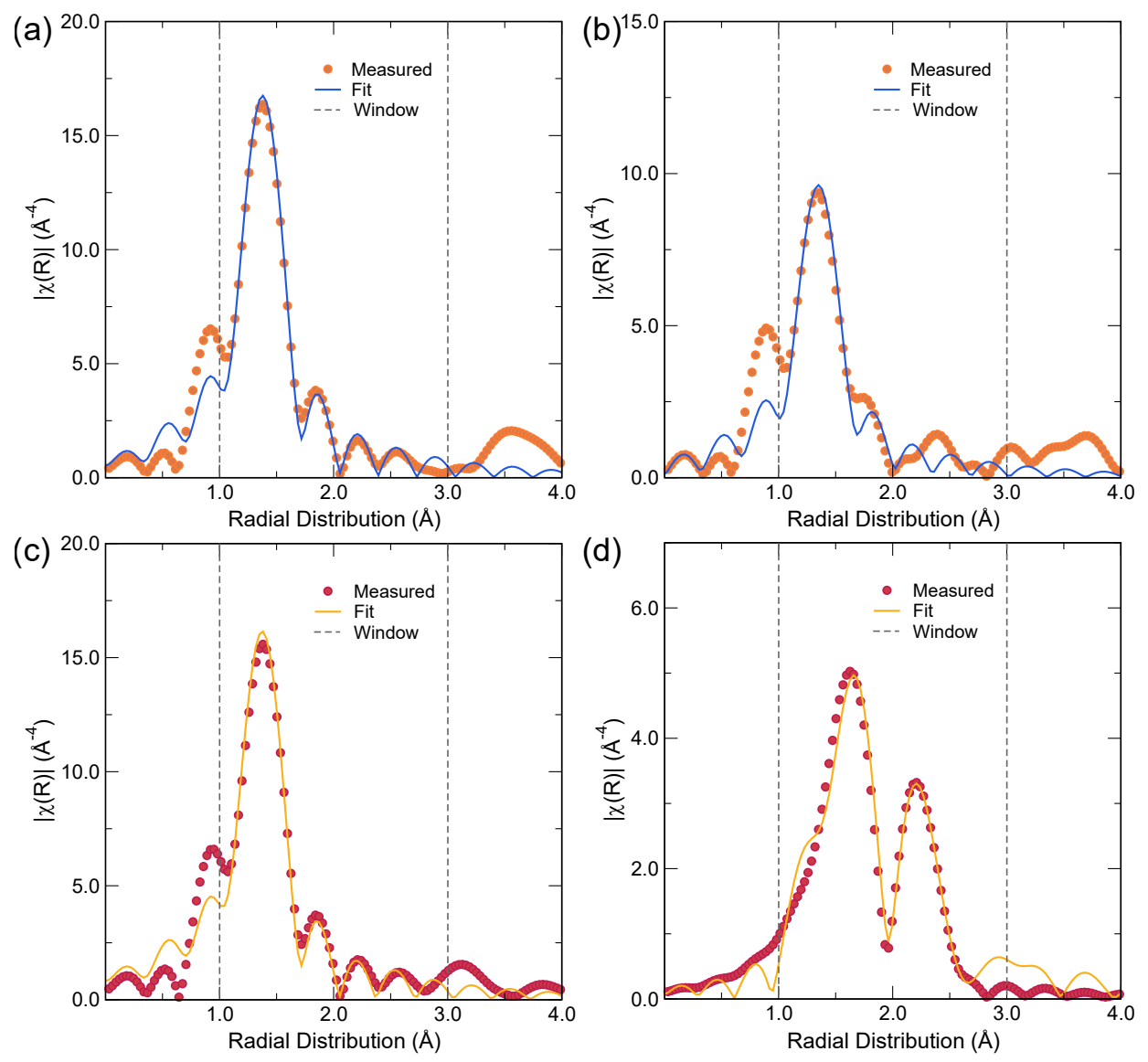

Figure S7: Fits of EXAFS radial distribution data for both $\mathrm{Y}_{2}\left(\mathrm{MoO}_{4}\right)_{3}$ and $\mathrm{Al}_{2}\left(\mathrm{MoO}_{4}\right)_{3}$ in their pristine $(\mathrm{a}, \mathrm{c})$ and fully lithiated states $(\mathrm{b}, \mathrm{d})$ using the models described in the text. 
Solid State NMR Measurements. Solid state NMR measurements of the ${ }^{27} \mathrm{Al},{ }^{7} \mathrm{Li}$, ${ }^{95} \mathrm{Mo}$, and ${ }^{89} \mathrm{Y}$ nuclei were used to provide information on local coordination environments and structural changes in $\mathrm{Al}_{2}\left(\mathrm{MoO}_{4}\right)_{3}$ and $\mathrm{Y}_{2}\left(\mathrm{MoO}_{4}\right)_{3}$.

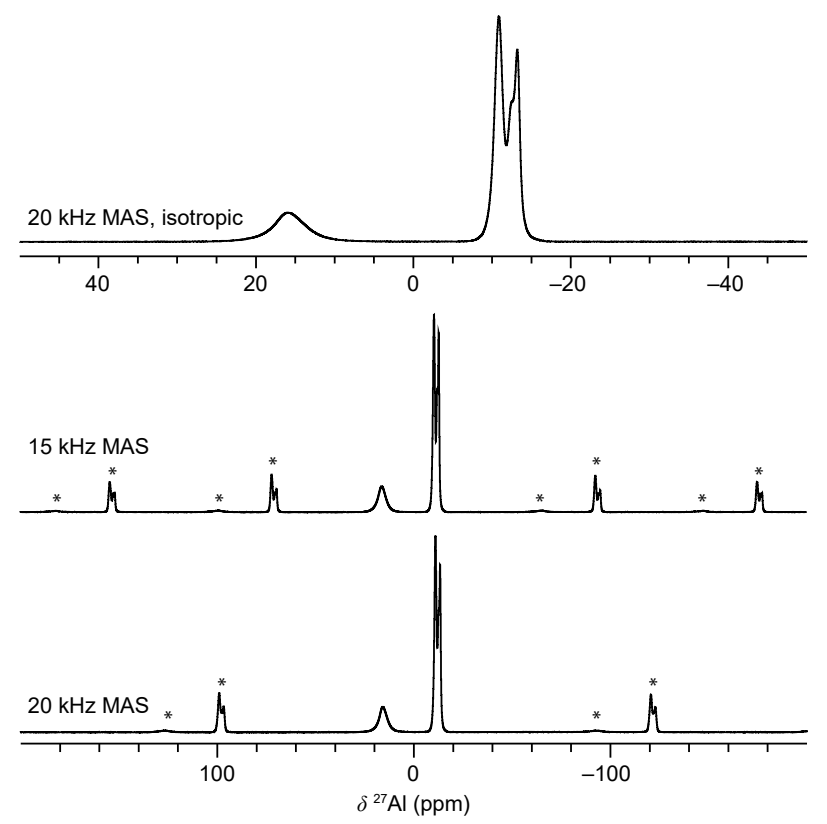

Figure S8: ${ }^{27} \mathrm{Al}$ solid-state NMR spectra of $\mathrm{Al}_{2}\left(\mathrm{MoO}_{4}\right)_{3}$ at different MAS speeds. Spinning sidebands move with MAS speed and are denoted with asterisks. Spectra were recorded at $16.4 \mathrm{~T}$.

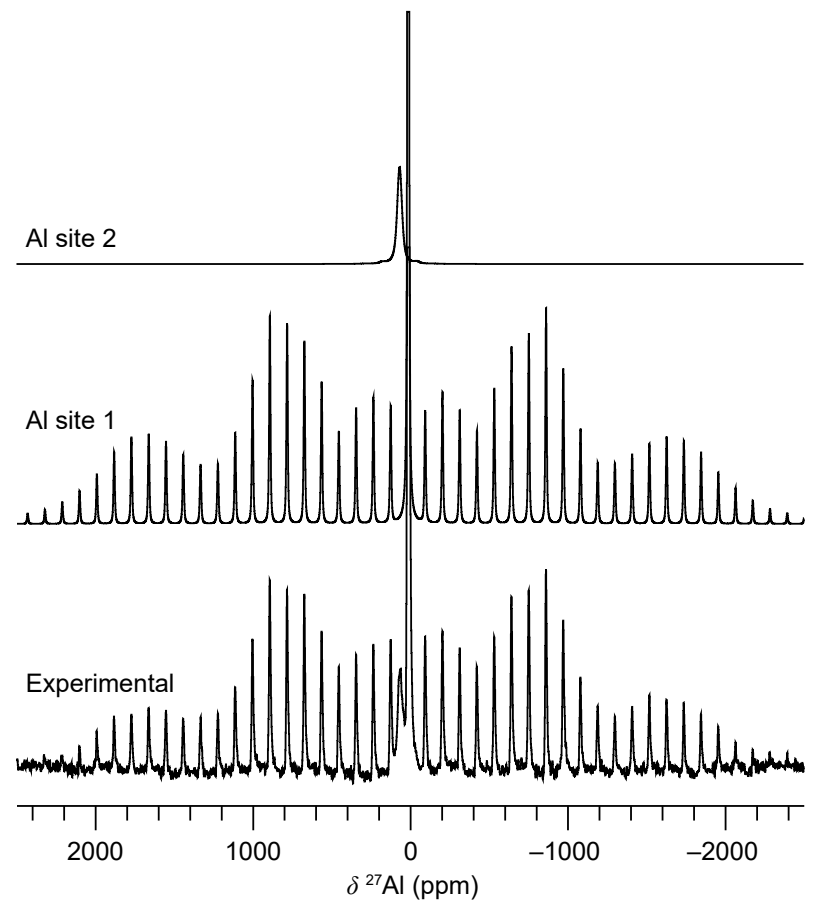

Figure S9: Fitted ${ }^{27} \mathrm{Al}$ NMR spectrum of $\mathrm{Li}_{5} \mathrm{Al}_{2}\left(\mathrm{MoO}_{4}\right)_{3}$ at $16.4 \mathrm{~T}$ and $20 \mathrm{kHz}$ MAS showing deconvolution into two sites. Site 1 , at $16.0 \pm 0.5 \mathrm{ppm}$, has a $C_{Q}$ of $2.4(1) \mathrm{MHz}$ and $\eta$ of $0.15(5)$ while site 2 , at $75 \pm 5 \mathrm{ppm}$, is broad with a large $C_{Q}$ of $6(1)$ Mhz. 


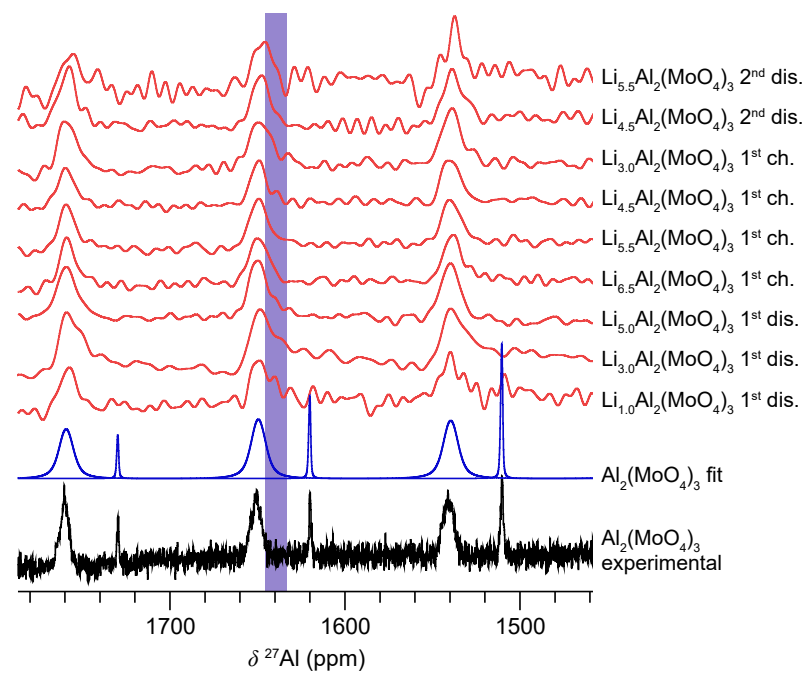

Figure S10: Al metal region of the ${ }^{27} \mathrm{Al} \mathrm{NMR}$ spectra of $\mathrm{Li}_{x} \mathrm{Al}_{2}\left(\mathrm{MoO}_{4}\right)_{3}$ during the first 1.5 discharge/charge cycles. The observed signals are spinning sidebands arising from the satellite transitions of the isotropic resonances of $\mathrm{Al}_{2}\left(\mathrm{MoO}_{4}\right)_{3}$, the $\mathrm{AlO}_{6}$-like defect, and $\mathrm{Li}_{x} \mathrm{Al}_{2}\left(\mathrm{MoO}_{4}\right)_{3}$ centered at $-12 \mathrm{ppm}, 16 \mathrm{ppm}$, and $14 \mathrm{ppm}$, respectively. The purple highlighted region denotes where the signal from Al metal would appear. Note that the intensity of these peaks represents $<0.5 \%$ of the total intensity (compare to Figure 5) and thus not even a subpercent Al metal signal is observed. Spectra were recorded at $16.4 \mathrm{~T}$ and $20 \mathrm{kHz}$ MAS.

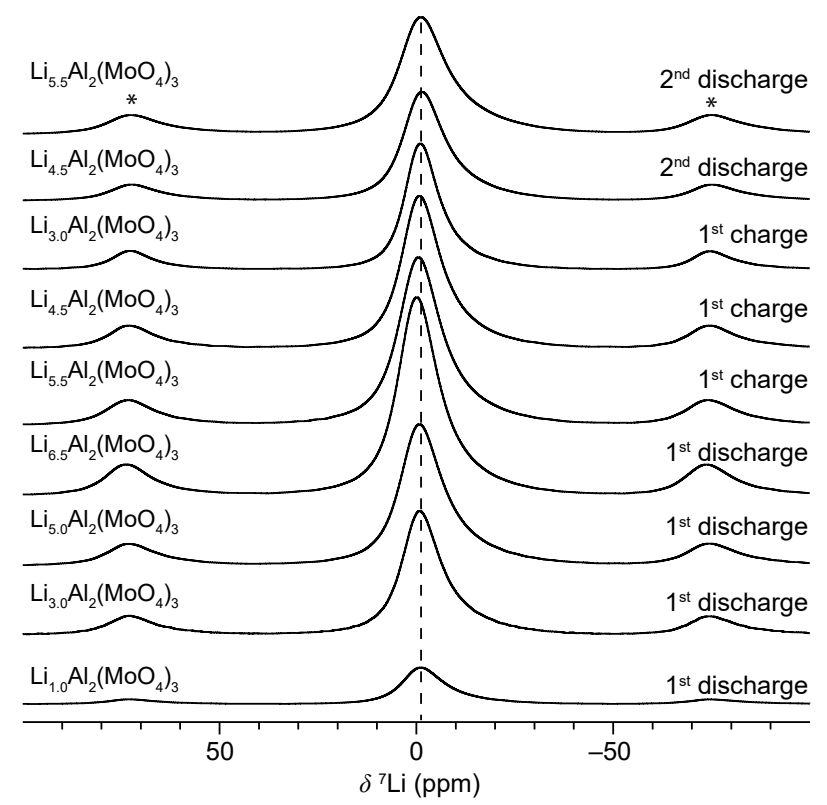

Figure S11: ${ }^{7} \mathrm{Li}$ NMR spectra of $\mathrm{Li}_{x} \mathrm{Al}_{2}\left(\mathrm{MoO}_{4}\right)_{3}$ during the first 1.5 discharge/charge cycles. No changes in shift or lineshape were observed as a function of state-of-charge nor as a function of $T_{1}$-filtering experiments with a short recycle delay (latter not shown). Spectra were recorded at $16.4 \mathrm{~T}$ and $20 \mathrm{kHz}$ MAS. 
To better characterize the structure, and out of interest in building our understanding of solid-state inorganic NMR, ${ }^{89} \mathrm{Y}$ and ${ }^{95} \mathrm{Mo}$ NMR spectra of the host structures were measured. ${ }^{89} \mathrm{Y}$ suffers from long relaxation times and thus it was not possible to record spectra on the relatively small amount of lithiated sample in a way that would have been analogous to ${ }^{27} \mathrm{Al}$ in Figure 5. In addition to the low receptivity of ${ }^{95} \mathrm{Mo}$, it is generally not possible to measure NMR spectra directly on a redox active transition metal outside of its diamagnetic state(s) due to extremely rapid paramagnetic relaxation.

The ${ }^{95} \mathrm{Mo}$ spectra of $\mathrm{Al}_{2}\left(\mathrm{MoO}_{4}\right)_{3}$ and $\mathrm{Y}_{2}\left(\mathrm{MoO}_{4}\right)_{3}$ and ${ }^{89} \mathrm{Y}$ spectrum of $\mathrm{Y}_{2}\left(\mathrm{MoO}_{4}\right)_{3}$ are expected to show six distinct Mo environments of equal population in $\mathrm{Al}_{2}\left(\mathrm{MoO}_{4}\right)_{3}$ and two Mo environments in a 2:1 ratio as well as a single $\mathrm{Y}$ environment in $\mathrm{Y}_{2}\left(\mathrm{MoO}_{4}\right)_{3}$. The ${ }^{95} \mathrm{Mo}$ spectrum of $\mathrm{Al}_{2}\left(\mathrm{MoO}_{4}\right)_{3}$ is consistent with the structure model in $P 2_{1} / a$ if the signals at -280 and $-325 \mathrm{ppm}$ are comprised of two overlapping quadrupolar resonances. The calculated shift range of $7 \mathrm{ppm}$ and $C_{Q}$ values of $0.7-1.8 \mathrm{MHz}$ are consistent with the spectrum. On the other hand, the ${ }^{95} \mathrm{Mo}$ and ${ }^{89} \mathrm{Y}$ spectra of $\mathrm{Y}_{2}\left(\mathrm{MoO}_{4}\right)_{3}$ suggest that multiple polymorphs or phases may be present because there are multiple ${ }^{89} \mathrm{Y}$ and ${ }^{95} \mathrm{Mo}$ signals. DFT calculations of the ${ }^{89} \mathrm{Y}$ chemical shielding $\mathrm{Y}_{2}\left(\mathrm{MoO}_{4}\right)_{3}$ were calibrated into a chemical shift by linear fitting of the shielding/shift relationship for 20 different yttrium environments in 12 yttrium-containing oxides, see Figure S14 and Table T1. The calculations predict an ${ }^{89} \mathrm{Y}$ chemical shift of -38.1 $\mathrm{ppm}$, in reasonable agreement with the strongest ${ }^{89} \mathrm{Y}$ resonance in Figure $\mathrm{S} 13$ at $-52 \mathrm{ppm}$.

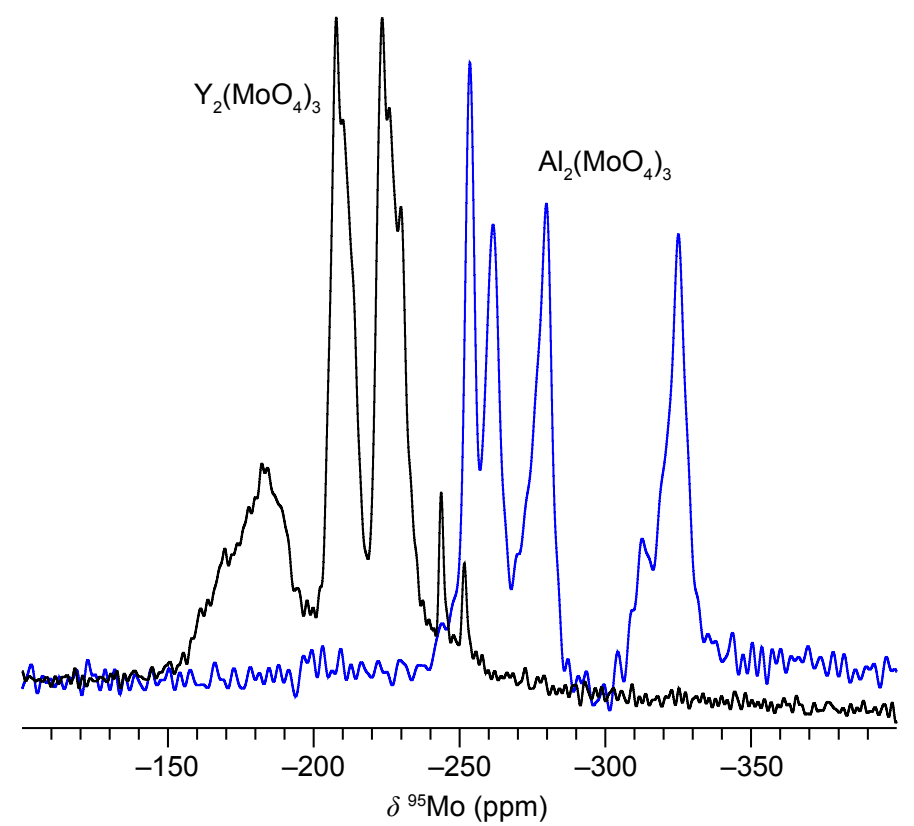

Figure S12: ${ }^{95} \mathrm{Mo}$ spectra of $\mathrm{Al}_{2}\left(\mathrm{MoO}_{4}\right)_{3}$ (blue) and $\mathrm{Y}_{2}\left(\mathrm{MoO}_{4}\right)_{3}$ (black) at $16.4 \mathrm{~T}$ and $12.5 \mathrm{kHz} \mathrm{MAS}$. 


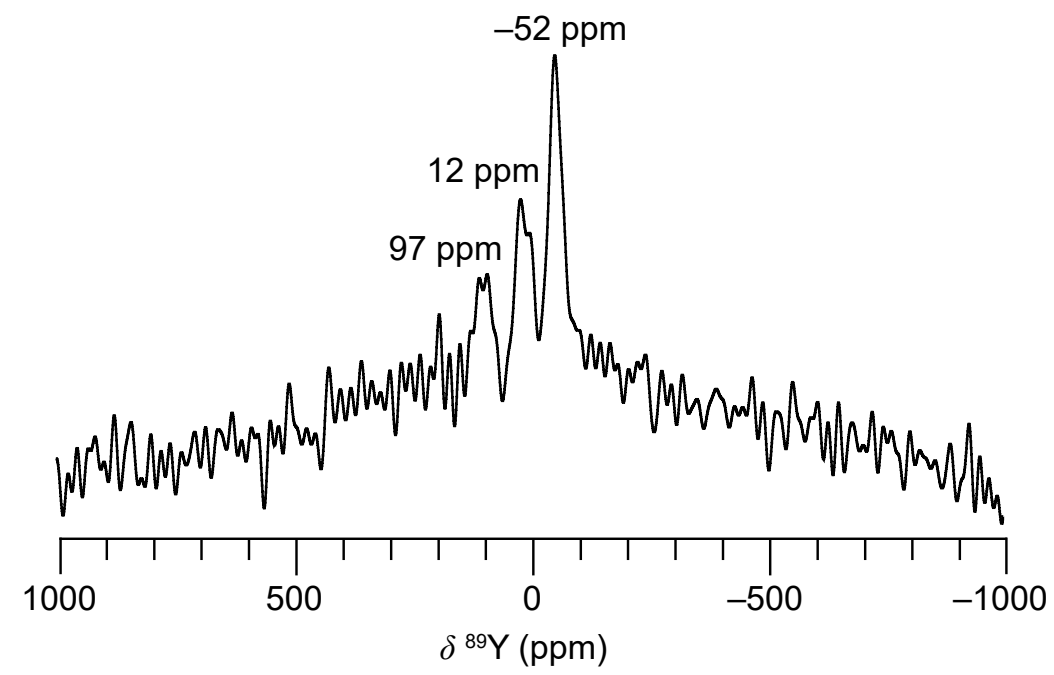

Figure S13: ${ }^{89} \mathrm{Y}$ spectrum of $\mathrm{Y}_{2}\left(\mathrm{MoO}_{4}\right)_{3}$ at $16.4 \mathrm{~T}$ and $12.5 \mathrm{kHz}$ MAS.

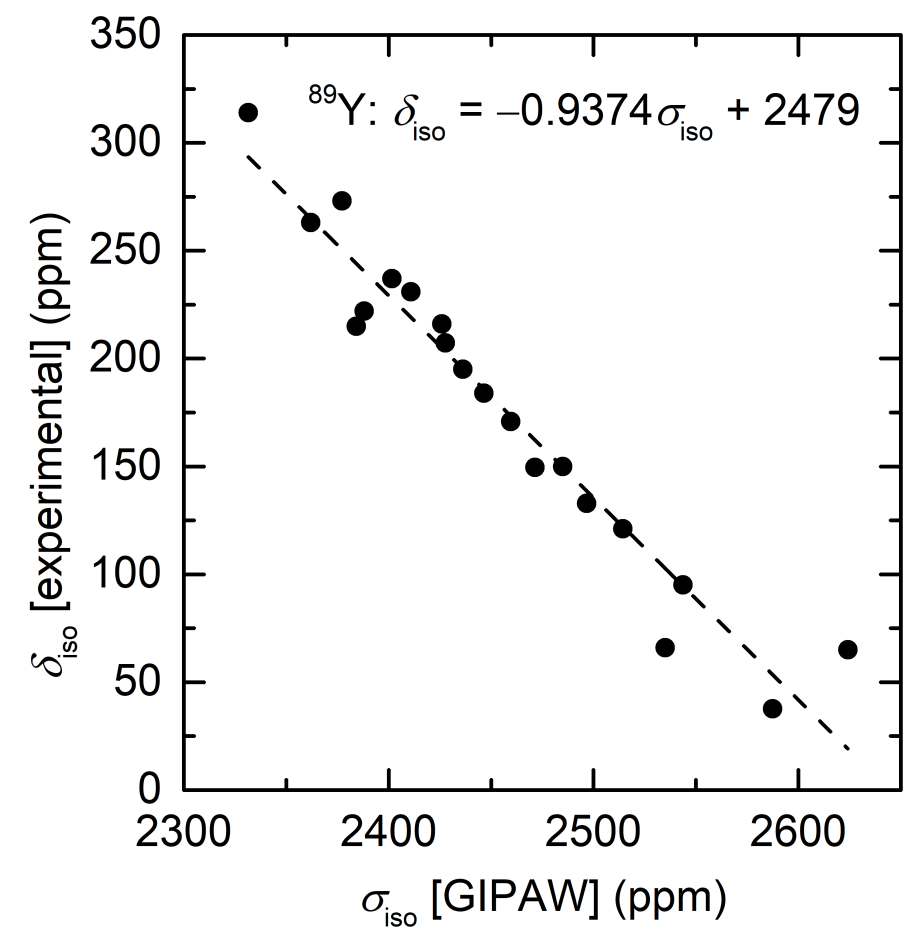

Figure S14: Experimental isotropic chemical shift $\left(\delta_{i s o}\right)$ vs. calculated isotropic chemical shielding $\left(\sigma_{i s o}\right)$ for 20 different ${ }^{89} \mathrm{Y}$ sites in the 12 yttrium oxide compounds listed in S.I. Table T1. 
Table T1: Table of ${ }^{89} \mathrm{Y}$ computed chemical shielding vs. experimental chemical shift for a series of yttrium oxide crystals.

\begin{tabular}{llllll}
\hline Compound & Space Group & ICSD No. & Site & $\sigma_{\text {iso }}(\mathrm{ppm})$ & $\delta_{\text {iso }}(\mathrm{ppm})$ \\
\hline $\mathrm{Y}_{2} \mathrm{O}_{3}$ & $I a \overline{3}$ & 66243 & 1 & 2331.55 & 314 \\
& & & 2 & 2377.17 & 273 \\
$\mathrm{Y}(\mathrm{OH})_{3}$ & $P 6_{3} / m$ & 200098 & 1 & 2535.03 & 66 \\
$\alpha-\mathrm{Y}_{2} \mathrm{Si}_{2} \mathrm{O}_{7}$ & $P \overline{1}$ & 164148 & 1 & 2496.64 & 132.9 \\
& & & 2 & 2543.70 & 95.1 \\
& & 3 & 2459.61 & 170.8 \\
& & 4 & 2587.46 & 37.7 \\
$\beta-\mathrm{Y}_{2} \mathrm{Si}_{2} \mathrm{O}_{7}$ & $C 2 / m$ & 281312 & 1 & 2427.69 & 207.3 \\
$\delta-\mathrm{Y}_{2} \mathrm{Si}_{2} \mathrm{O}_{7}$ & $P n a m$ & 33721 & 1 & 2514.29 & 121.1 \\
$\mathrm{Y}_{2} \mathrm{Sn}_{2} \mathrm{O}_{7}$ & $F d \overline{3} m Z$ & 74706 & 1 & 2485.04 & 150 \\
$\mathrm{Y}_{2} \mathrm{Ti}_{2} \mathrm{O}_{7}$ & $F d \overline{3} m Z$ & 14242 & 1 & 2624.15 & 65 \\
$\mathrm{Y}_{4} \mathrm{Al}_{2} \mathrm{O}_{9}$ & $P 21 / c$ & 51076 & 1 & 2436.30 & 195 \\
& & & 2 & 2410.92 & 231 \\
& & 3 & 2446.47 & 184 \\
$\mathrm{YAlO}_{3}$ & $P n m a$ & 4115 & 1 & 2426.03 & 216 \\
$\chi-\mathrm{Y}_{2} \mathrm{SiO}_{5}$ & $I 2 / a$ & 28021 & 1 & 2401.18 & 215 \\
$\mathrm{Y}_{3} \mathrm{Al}_{5} \mathrm{O}_{12}$ & $I a \overline{3} d$ & 20090 & 2 & 2471.41 & 149.5 \\
$\mathrm{YScO}_{3}$ & $P b n m$ & 237285 & 1 & 2388.06 & 222 \\
\hline
\end{tabular}

Octahedral Angles The angles of the octahedral subunits demonstrated in 1 are provided in the following table.

We note the distorted nature of the octahedra create multiple distinct angles.

Table T2: Table of octahedral angles for $\mathrm{Y}_{2}\left(\mathrm{MoO}_{4}\right)_{3}$ and $\mathrm{Al}_{2}\left(\mathrm{MoO}_{4}\right)_{3}$ to demonstrate the distortions present in the octahedral subunits.

\begin{tabular}{lcc}
\hline Bond & $\mathrm{Y}_{2}\left(\mathrm{MoO}_{4}\right)_{3}$ & $\mathrm{Al}_{2}\left(\mathrm{MoO}_{4}\right)_{3}$ \\
\hline O2-M-O1 & $90.91^{\circ}$ & $93.52^{\circ}$ \\
O2-M-O3 & $86.22^{\circ}$ & $86.60^{\circ}$ \\
O2-M-O4 & $89.97^{\circ}$ & $89.51^{\circ}$ \\
O2-M-O5 & $88.69^{\circ}$ & $88.18^{\circ}$ \\
O6-M-O1 & $89.32^{\circ}$ & $87.48^{\circ}$ \\
O6-M-O3 & $94.73^{\circ}$ & $92.69^{\circ}$ \\
O6-M-O4 & $89.66^{\circ}$ & $94.18^{\circ}$ \\
O6-M-O5 & $90.36^{\circ}$ & $87.61^{\circ}$ \\
\hline
\end{tabular}


Computational Band Diagrams of $\mathrm{Y}_{2}\left(\mathrm{MoO}_{4}\right)_{3}$ and $\mathrm{Al}_{2}\left(\mathrm{MoO}_{4}\right)_{3}$. Density functional theory was used to calculate the band diagrams of $\mathrm{Y}_{2}\left(\mathrm{MoO}_{4}\right)_{3}$ and $\mathrm{Al}_{2}\left(\mathrm{MoO}_{4}\right)_{3}$ as shown in Figure S15. Both materials possess large band gaps on the order of $5 \mathrm{eV}$, with flat bands suggestive of low electron mobility. The introduction of $\mathrm{Y} 4 d$ states in $\mathrm{Y}_{2}\left(\mathrm{MoO}_{4}\right)_{3}$ increases the covalency of the material in comparison to $\mathrm{Al}_{2}\left(\mathrm{MoO}_{4}\right)_{3}$.
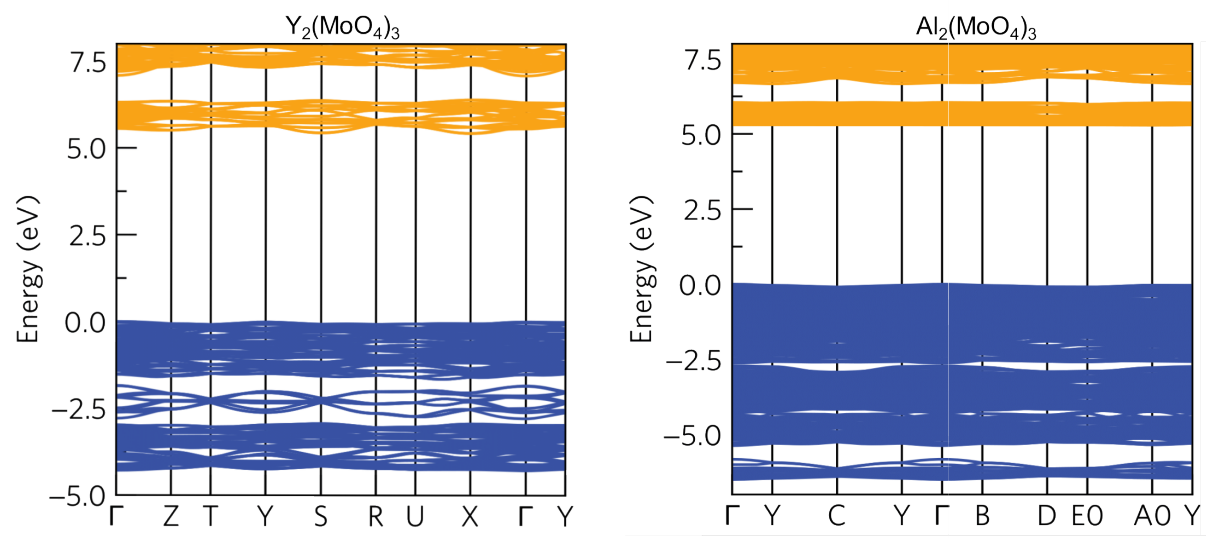

Figure S15: The calculated band diagrams of both $\mathrm{Y}_{2}\left(\mathrm{MoO}_{4}\right)_{3}$ and $\mathrm{Al}_{2}\left(\mathrm{MoO}_{4}\right)_{3}$.

Geometry Optimization and Brillouin Zones. Structural optimisations were deemed converged when the sum of all forces on each atom totalled less than $10 \mathrm{meV} \AA^{-1}$. The lattice parameters of $\mathrm{Al}_{2}\left(\mathrm{MoO}_{4}\right)_{3}$ and $\mathrm{Y}_{2}\left(\mathrm{MoO}_{4}\right)_{3}$ optimised using the PBEsol functional are provided in Table T3. In general, the results show good agreement with experiment, with all values within $1 \%$ of the experimentally determined parameters.

Table T3: Lattice parameters for $\mathrm{Al}_{2}\left(\mathrm{MoO}_{4}\right)_{3}$ and $\mathrm{Y}_{2}\left(\mathrm{MoO}_{4}\right)_{3}$ calculated using the PBEsol functional. Difference versus experiment in percent given in parentheses

\begin{tabular}{lcccccc}
\hline Compound & $a / \AA$ & $b / \AA$ & $c / \AA$ & $\alpha /{ }^{\circ}$ & $\beta /{ }^{\circ}$ & $\gamma /{ }^{\circ}$ \\
\hline $\mathrm{Al}_{2}\left(\mathrm{MoO}_{4}\right)_{3}$ & $15.472(+0.60)$ & $9.090(+0.49)$ & $17.959(+0.40)$ & $90(0)$ & $125.3(-0.04)$ & $90(0)$ \\
$\mathrm{Y}_{2}\left(\mathrm{MoO}_{4}\right)_{3}$ & $14.001(+0.96)$ & $9.989(+0.55)$ & $10.103(+0.82)$ & $90(0)$ & $90(0)$ & $90(0)$ \\
\hline
\end{tabular}

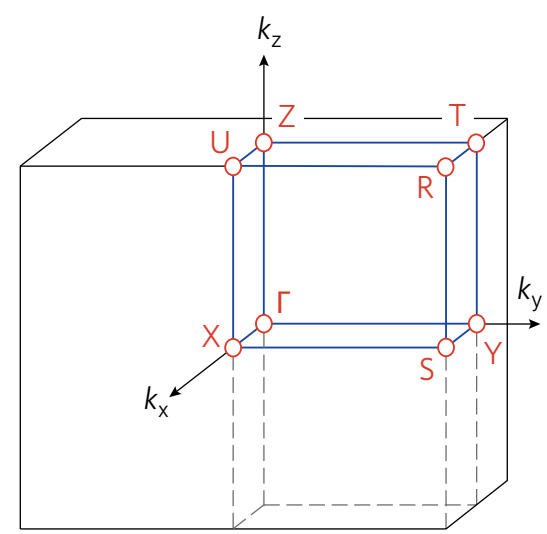

Figure S16: Brillouin zone for the Pbcn space group, indicating all high-symmetry $k$-points. The coordinates of the high symmetry $k$-points are: $\Gamma=(0,0,0) ; \mathrm{Y}=\left(\frac{1}{2}, 0,0\right) ; \mathrm{X}=\left(0, \frac{1}{2}, 0\right) ; \mathrm{Z}=\left(0,0, \frac{1}{2}\right) ; \mathrm{U}=\left(0, \frac{1}{2}, \frac{1}{2}\right) ; \mathrm{T}=\left(\frac{1}{2}, 0, \frac{1}{2}\right) ; \mathrm{S}$ $=\left(\frac{1}{2}, \frac{1}{2}, 0\right) ; \mathrm{R}=\left(0, \frac{1}{2}, \frac{1}{2}\right)$ 


\section{References}

(1) Borkiewicz, O. J.; Shyam, B.; Wiaderek, K. M.; Kurtz, C.; Chupas, P. J.; Chapman, K. W. The AMPIX electrochemical cell: a versatile apparatus for in situ X-ray scattering and spectroscopic measurements. $J$. Appl. Crystallogr. 2012, 45, 1261-1269.

(2) Toby, B. H.; Dreele, R. B. V. GSAS-II: the genesis of a modern open-source all purpose crystallography software package. J. Appl. Crystallogr. 2013, 46, 544-549.

(3) Ravel, B.; Newville, M. ATHENA, ARTEMIS, HEPHAESTUS: data analysis for X-ray absorption spectroscopy using IFEFFIT. J. Synchrotron Radiat. 2005, 12, 537-541.

(4) Ankudinov, A. L.; Ravel, B.; Rehr, J. J.; Conradson, S. D. Real-space multiple-scattering calculation and interpretation of x-ray-absorption near-edge structure. Phys. Rev. B 1998, 58, 7565-7576.

(5) Clark, S. J.; Segall, M. D.; Pickard, C. J.; Hasnip, P. J.; Probert, M. I. J.; Refson, K.; Payne, M. C. First principles methods using CASTEP. Z. Kristallogr. Cryst. Mater. 2005, 220.

(6) Perdew, J. P.; Burke, K.; Ernzerhof, M. Generalized Gradient Approximation Made Simple. Phys. Rev. Lett. 1996, $77,3865-3868$.

(7) Marinkovic, B.; Jardim, P.; de Avillez, R.; Rizzo, F. Negative thermal expansion in $\mathrm{Y}_{2} \mathrm{Mo}_{3} \mathrm{O}_{12}$. Solid State Sci. 2005, 7, 1377-1383.

(8) Harrison, W.; Cheetham, A.; Faber, J. The crystal structure of aluminum molybdate, $\mathrm{Al}_{2}\left(\mathrm{MoO}_{4}\right)_{3}$, determined by time-of-flight powder neutron diffraction. J. Solid State Chem. 1988, 76, 328-333.

(9) Vanderbilt, D. Soft self-consistent pseudopotentials in a generalized eigenvalue formalism. Phys. Rev. B 1990, 41, 7892-7895.

(10) Monkhorst, H. J.; Pack, J. D. Special points for Brillouin-zone integrations. Phys. Rev. B 1976, 13, 5188-5192.

(11) Kresse, G.; Hafner, J. Ab Initio Molecular Dynamics for Liquid Metals. Phys. Rev. B 1993, 47, 558-561.

(12) Kresse, G.; Hafner, J. Ab Initio Molecular-Dynamics Simulation of the Liquid-Metal Amorphous-Semiconductor Transition in Germanium. Phys. Rev. B 1994, 49, 14251-14269.

(13) Kresse, G.; Furthmüller, J. Efficient Iterative Schemes for Ab Initio Total-Energy Calculations Using a PlaneWave Basis Set. Phys. Rev. B 1996, 54, 11169-11186.

(14) Kresse, G.; Furthmüller, J. Efficiency of Ab Initio Total Energy Calculations for Metals and Semiconductors Using a Plane Wave Basis Set. Comput. Mater. Sci. 1996, 6, 15. 
(15) Perdew, J. P.; Ruzsinszky, A.; Csonka, G. I.; Vydrov, O. a.; Scuseria, G. E.; Constantin, L. a.; Zhou, X.; Burke, K. Restoring the Density-Gradient Expansion for Exchange in Solids and Surfaces. Phys. Rev. Lett. 2008, 100, 136406 .

(16) Bashian, N. H.; Zhou, S.; Zuba, M.; Ganose, A. M.; Stiles, J. W.; Ee, A.; Ashby, D. S.; Scanlon, D. O.; Piper, L. F. J.; Dunn, B.; Melot, B. C. Correlated Polyhedral Rotations in the Absence of Polarons during Electrochemical Insertion of Lithium in $\mathrm{ReO}_{3}$. ACS Energy Lett. 2018, 3, 2513-2519.

(17) Regoutz, A. et al. Insights into the electronic structure of $\mathrm{OsO}_{2}$ using soft and hard x-ray photoelectron spectroscopy in combination with density functional theory. Phys. Rev. Materials 2019, 3, 025001.

(18) Ganose, A. M.; Jackson, A. J.; Scanlon, D. O. sumo: Command-line tools for plotting and analysis of periodic ab initio calculations. J. Open Source Softw. 2018, 3, 717.

(19) Krukau, A. V.; Vydrov, O. A.; Izmaylov, A. F.; Scuseria, G. E. Influence of the Exchange Screening Parameter on the Performance of Screened Hybrid Functionals. J. Chem. Phys. 2006, 125, 224106. 